\title{
Cyclic electron flow provides acclimatory plasticity for the photosynthetic machinery under various environmental conditions and developmental stages
}

\author{
Marjaana Suorsa * \\ Molecular Plant Biology, Department of Biochemistry, University of Turku, Turku, Finland
}

Photosynthetic electron flow operates in two modes, linear and cyclic. In cyclic electron flow (CEF), electrons are recycled around photosystem I. As a result, a transthylakoid proton gradient $(\Delta \mathrm{pH})$ is generated, leading to the production of ATP without concomitant production of $\mathrm{NADPH}$, thus increasing the ATP/NADPH ratio within the chloroplast. At least two routes for CEF exist: a PROTON GRADIENT REGULATION5-PGRL1-and a chloroplast NDH-like complex mediated pathway. This review focuses on recent findings concerning the characteristics of both CEF routes in higher plants, with special emphasis paid on the crucial role of CEF in under challenging environmental conditions and developmental stages.

Wah Soon Chow,

Edited by:

Wei Huang

Kunming Institute of Botany, Chinese

Academy of Sciences, China

Reviewed by:

The Australian National University,

Australia

Jean Alric,

Centre National de la Recherche

Scientifique, France

*Correspondence:

Marjaana Suorsa,

Molecular Plant Biology, Department of Biochemistry, University of Turku,

Fl-20014 Turku, Finland

msuorsa@utu.fi

Specialty section:

This article was submitted to

Plant Physiology,

a section of the journal

Frontiers in Plant Science

Received: 04 August 2015 Accepted: 14 September 2015

Published: 28 September 2015

Citation:

Suorsa M (2015) Cyclic electron flow provides acclimatory plasticity

for the photosynthetic machinery

under various environmental

conditions and developmental stages.

Front. Plant Sci. 6:800.

doi: 10.3389/fp/s.2015.00800

Keywords: acclimation, cyclic electron flow, development, electron transfer, environment, NDH complex, PGR5, PGRL1

\section{Introduction}

Photosynthetic light reactions occur in four thylakoid membrane-embedded multiprotein complexes; photosystem (PS) II, cytochrome $b_{6} f\left(C y t b_{6} f\right)$, PSI and ATP synthase. In higher plants, these complexes are unevenly distributed along thylakoid membrane, as PSII complexes are mostly located in grana stacks, whilst PSI and the ATP synthase are enriched in the stroma-exposed thylakoids. The Cyt $b_{6} f$ complex has been traditionally assigned to be rather evenly distributed between the appressed and non-appressed regions, however, recent results from biochemical (Grieco et al., 2015) and immunolabeling (Armbruster et al., 2013) experiments suggest its predominant localization in the non-appressed thylakoid domains.

In linear electron flow (LEF), the PSs function in series and electrons are transferred all the way from water to NADP+ with concomitant production of NADPH and ATP. Cyclic electron flow (CEF), in contrast, recycles electrons around PSI by re-routing them from ferredoxin $(\mathrm{Fd})$ to the plastoquinone $(\mathrm{PQ})$. As a result, a transthylakoid proton gradient $(\triangle \mathrm{pH})$ is generated, leading to the production of only ATP. Consequently, CEF has been proposed to balance the ATP/NAPDH ratio. The $\Delta \mathrm{pH}$ component forms part of the proton motive force (pmf), which drives the ATP synthase and can be monitored as electrochromic bandshift (ECS) from intact leaves. Besides $\Delta \mathrm{pH}$, also the transthylakoid electric potential $(\Delta \psi)$ contributes to pmf. The transthylakoid $\Delta \mathrm{pH}$ is crucial for the regulation of LEF via two mechanisms: (i) increased $\Delta \mathrm{pH}$ downregulates the Cyt $b_{6} f$ complex ("photosynthetic control"), 
which limits the electron flow from PSII toward PSI and thus protects PSI particularly upon sudden exposure to high light intensity (Joliot and Johnson, 2011; Suorsa et al., 2012). Furthermore, (ii) lumen acidification triggers induction of nonphotochemical quenching (NPQ) of excess light energy, which protects PSII from photoinhibition (Li et al., 2009).

Two routes for CEF exist: (i) antimycin A sensitive pathway, which includes the PROTON GRADIENT REGULATION5 (PGR5) and PGR5-LIKE PROTEIN1 (PGRL1) proteins (Munekage et al., 2002, 2004; DalCorso et al., 2008) and (ii) antimycin A insensitive pathway, which transfers electrons via the $\mathrm{NAD}(\mathrm{P}) \mathrm{H}$ dehydrogenase-like $(\mathrm{NDH})$ complex (Ifuku et al., 2011) [for a recent reviews, see (Leister and Shikanai, 2013; Shikanai, 2014]. Both of these pathways receive electrons from Fd (Yamamoto et al., 2011; Hertle et al., 2013). It has been estimated that in C3 plants, roughly one tenth of the total electron flow derives from CEF (Avenson et al., 2005). However, it is likely that under some specific conditions, such as upon onset on light illumination and under certain environmental and developmental conditions (see below), the proportion of CEF might be substantially higher. Based on the current knowledge, the major part of CEF in C3 plants is thought to be driven by the PGR5-PGRL1 pathway (Avenson et al., 2005; Wang et al., 2014). Besides defective pmf, the pgr5 mutants of both Arabidopsis and rice have also been shown to exhibit an elevated proton conductance of the ATP synthase (Avenson et al., 2005; Nishikawa et al., 2012; Wang et al., 2014), as well as an increased amount of the ATP synthase $\beta$ subunit (Suorsa et al., 2012). In contrast, the $n d h$ mutants show an ATP synthase activity similar to that of wild type (WT; Wang et al., 2014). Whilst a deficiency of either of CEF pathways does not lead to a visual phenotype under standard growth conditions, a complete inhibition of CEF in the Arabidopsis pgr5 crr-2 double mutant deficient in both pathways severely impairs plant growth and performance (Munekage et al., 2004), implying that CEF is essential for photosynthesis even in the C3 species.

This review focuses on the recent findings concerning the characteristics of both CEF routes, with special emphasis paid on the crucial role of CEF in higher plants under challenging environmental and developmental conditions.

\section{The PGR5-PGRL1 Route of CEF}

The crucial role of the PGR5 protein for the maintenance of proper $\Delta \mathrm{pH}$ was demonstrated already more than 10 years ago (Munekage et al., 2002) and a few years later, its interaction with PGRL1 was characterized (DalCorso et al., 2008). Whilst PGR5 does not contain a transmembrane helix, it is present in purified thylakoid fractions (Munekage et al., 2002). The interaction of PGR5 with the membrane-spanning PGRL1 likely takes place via conserved Cys residues present in both PGR5 and PGRL1 (Hertle et al., 2013). In vitro -assays indicate that PGRL1 interacts also with Cytb6f, Fd, the PSI subunit PsaD, and with both isoforms of the $\mathrm{Fd}^{-N A D P}{ }^{+}$-oxidoreductase (FNR) (DalCorso et al., 2008). Furthermore, it was recently demonstrated that the PGRL1-PGR5 complex is capable of accepting electrons from
Fd, and PGRL1 can reduce quinones, indicating that the longsought hypothetical ferredoxin-plastoquinone reductase (FQR) has finally been experimentally characterized (Hertle et al., 2013).

PROTON GRADIENT REGULATION5 is present in all photosynthetic organisms, whereas PGRL1 is specific for green algae and plants (DalCorso et al., 2008). In the green algae Chlamydomonas reinhardtii (Chlamydomonas), PGRL1 has been shown to be present in the protein complex mediating CEF under state 2 conditions, together with PSI, its light harvesting complex (LHCI), LHCII, Cyt $b_{6} f$, and FNR (Iwai et al., 2010). PGR5, in contrast, was not found to be part of the complex (Iwai et al., 2010). However, the recently characterized Chlamydomonas pgr5 and pgrl1 mutants (Petroutsos et al., 2009; Tolleter et al., 2011; Dang et al., 2014; Johnson et al., 2014) exhibit characteristics resembling Arabidopsis pgr5 and pgrl1 mutants, indicating that also the PGR5 protein of Chlamydomonas has a role in CEF.

In Arabidopsis, PGRL1 is encoded by two genes, the PGRL1A isoform (encoded by the AT4G22890 gene) of which has been shown to be phosphorylated by the STN8 kinase (Reiland et al., 2011). Even though the stn 8 mutant was capable of reaching similar overall rate of CEF as compared to WT, its capacity to maintain CEF upon shift from darkness to light was lowered (Reiland et al., 2011), indicating that the STN8 kinase regulates the kinetics of CEF. However, as not only green algae, diatoms, mosses, and lycophytes, but also the monocot species lack the phosphorylated threonine from their PGRL1A sequence, this regulatory mechanism seems to be specific for dicots (Reiland et al., 2011). Thus, evolutionary aspects should be taken into account before drawing strict conclusions about the regulation of CEF (see also below).

\section{The Chloroplast NDH Complex}

The chloroplast NDH complex is located in non-appressed thylakoid membranes, and is present in small amounts as compared to major photosynthetic protein complexes. Besides functioning in $\mathrm{CEF}, \mathrm{NDH}$ is considered to be involved also in chlororespiration (Rumeau et al., 2007). In Arabidopsis, the chloroplast NDH complex is composed of more than 30 subunits, which form five subcomplexes: the membraneembedded subcomplex, the subcomplexes A and B, the electron donor subcomplex and the lumenal subcomplex [for recent reviews, see (Peng et al., 2010; Ifuku et al., 2011)]. The electron donor subcomplex is made of the subunits NdhS, T, U, and V (Yamamoto et al., 2011; Fan et al., 2015), of which the NdhS subunit is responsible for the binding of Fd (Yamamoto et al., 2011; Yamamoto and Shikanai, 2013). The subcomplexes $B$ and the lumenal subcomplex are absent from cyanobacteria. Furthermore, also a liverworth Marchantia polymorpha lacks the lumenal subcomplex (Ueda et al., 2012). In angiosperms, the NDH complex further forms supercomplexes with PSI, the minor LHCI proteins Lhac5 and Lhca6 functioning as linkers (Peng et al., 2008, 2009; Peng and Shikanai, 2011; Kouril et al., 2014), whereas in cyanobacteria and Marchantia, NDH exists as a single complex. Intriguingly, gymnosperms and Chlamydomonas lack the chloroplast NDH complex (Wakasugi et al., 1994; Maul et al., 
2002), however, in Chlamydomonas, a type-II NDH (NDH-2) has been shown to function in the non-photochemical PQ reduction (Mus et al., 2005; Jans et al., 2008; Desplats et al., 2009).

While the NDH complex plays a crucial role for carbon fixation in the bundle sheath cells of the C4 plants (Majeran et al., 2008), the physiological role of the NDH complex in mature C3 plants has remained largely uncharacterized. However, existence and maintenance of such a massive complex with both nuclear and plastid encoded subunits and a complex assembly pathway (Peng et al., 2009) concomitantly suggest that the NDH complex must bear a crucial role for plant performance. Particularly the drastic phenotype of the pgr5 crr-2 double mutant even under optimal conditions (Munekage et al., 2004) indicates that the $\mathrm{NDH}$-mediated CEF bears a compensatory role in the pgr5 mutant background. The exact molecular mechanism for this still remains elusive, particularly as no increase in the level of the $\mathrm{NDH}$ subunits has been reported for the pgr5 mutant (Munekage et al., 2004; DalCorso et al., 2008; Suorsa et al., 2012). However, it has been suggested that NDH might act as a proton pump (Shikanai, 2014), similar to mitochondrial complex I (Baradaran et al., 2013). The role for NDH in protonpumping and lumen acidification would explain the severe phenotype of pgr5 $\mathrm{cr}-2$ double mutant. However, experimental evidence demonstrating such a function for $\mathrm{NDH}$ is still missing.

The present lack of physiological knowledge on the role of $\mathrm{NDH}$ complex likely stems from the fact that majority of the studies concerning the NDH complex have been focused on identification and characterization of the novel subunits and assembly factors, rather than in addressing its functional role under varying environmental and/or developmental conditions. It is highly likely that during the forthcoming years, our knowledge on the physiological role of NDH will be markedly broadened. Indeed, the rice $n d h$ mutants were recently shown to exhibit disturbed electron transfer parameters as well as reduced growth and yield particularly under low light conditions, highlighting the physiological significance of the $\mathrm{NDH}$ complex under non-optimal growth conditions (Yamori et al., 2015).

The involvement of NDH also in chloroplast redox regulation is already well-documented, and it is conceivable that the $\mathrm{NDH}$-dependent $\mathrm{CEF}$ has a role in alleviating oxidative stress under various challenging conditions, such as under drought, extreme temperatures or during early developmental phases (see below). It has been shown that treatment of barley leaves with $\mathrm{H}_{2} \mathrm{O}_{2}$ increased the expression of plastid-encoded $\mathrm{NDH}$ genes and subunits (Casano et al., 2001). It was also recently demonstrated that increased levels of $\mathrm{H}_{2} \mathrm{O}_{2}$, occurring either after infiltration of the WT Arabidopsis leaves or in mutants producing elevated levels of $\mathrm{H}_{2} \mathrm{O}_{2}$, triggered specifically the $\mathrm{NDH}$-dependent CEF (Strand et al., 2015). In agreement with these observations, Arabidopsis ndh mutants showed enhanced levels of foliar $\mathrm{H}_{2} \mathrm{O}_{2}$ upon transfer of plants from darkness to light (Sirpio et al., 2009). On the other hand, Arabidopsis mutants with strongly lowered production of glutathione or thylakoid-bound ascorbate peroxidase have been shown to downregulate the expression of several nuclear genes encoding the NDH subunits or assembly factors (Queval and Foyer, 2012).

Arabidopsis ndh mutants have been demonstrated to exhibit increased resistance against fungal pathogen (Garcia-Andrade et al., 2013). Vice versa, inoculation of the WT plants with fungal pathogens or chitosan, a pathogen-associated molecular pattern that triggers immune responses, resulted in rapid and specific decline in the content of the NDH complex subunit NdhI. More specifically, pathogen exposure was shown to include modulated editing of the chloroplast-encoded NDH transcripts (Garcia-Andrade et al., 2013). The chloroplast NDH complex thus appears to be involved in plant immunity regulation. Intriguingly, an extrinsic PSII oxygen evolving complex protein PsbQ has been shown to be a specific target for pathogen attack (Rodriguez-Herva et al., 2012), and the lumenal subcomplex of NDH contains proteins homologous to PsbQ (Suorsa et al., 2010; Yabuta et al., 2010). However, it still remains to be elucidated whether some subunits or subcomplex(es) of $\mathrm{NDH}$ bear more important role in immune responses than others.

\section{The Role of PGR5 in Acclimation to Fluctuating Light Intensities}

Involvement of the PGR5 protein in acclimation to high light has been demonstrated already upon initial characterization of the protein, as the pgr5 mutants were found to be more susceptible to high light intensities as compared to WT (Munekage et al., 2002). The pgr5 mutant is incapable of the induction of the NPQ; furthermore, and in contrast to the npq4 mutant (Grieco et al., 2012; Tikkanen et al., 2015), pgr5 cannot oxidize its P700 under high light (Munekage et al., 2002). Indeed, high light intensities lead to preferential damage of PSI in pgr5 plants (Munekage et al., 2002), confirming a crucial role of the PGR5 protein in photoprotection of PSI under high light. In line with its susceptibility for high light, it has been reported that the pgr5 mutant shows stunted growth under high light intensities, a defect alleviated by increased CO2 concentrations (Munekage et al., 2008).

In any case, it is remarkable that the pgr5 mutant is capable of growing under constant high light, since growth under fluctuating light, in which low light intensity of $50 \mu \mathrm{mol}$ photons $\mathrm{m}^{-2} \mathrm{~s}^{-1}$ becomes repeatedly interrupted with peaks of moderately high light $\left(500 \mu \mathrm{mol}\right.$ photons $\left.\mathrm{m}^{-2} \mathrm{~s}^{-1}\right)$, resulted in lethal phenotype of the pgr5 mutant (Tikkanen et al., 2010; Suorsa et al., 2012). In order to successfully acclimate for fluctuating light intensity, plants must acquire rapid acclimation shifts between an intense light harvesting mode (low light phase) and a quenching mode (high light phase). Consequently, long-term acclimation strategies applied for growth under constant high light, such as leaf pigmentation, morphological, and ultrastructural changes and constantly enhanced NPQ (Li et al., 2009), cannot be utilized for acclimation for the high light phases of fluctuating light, as they would severely compromise efficient light harvesting under low light phases. Therefore, acclimation to fluctuating light requires specific acclimation mechanisms, of which the so 
called "photosynthetic control," i.e., strong down-regulation of electron flow to PSI, bears the most crucial role (for review, Suorsa et al., 2013). Limitation of electron flow to PSI enables its oxidation upon high light phases of the fluctuating light, thus safeguarding the function and stability of PSI. Indeed, Arabidopsis pgr5 mutants exhibit severe photoinhibition of PSI under fluctuating light (Suorsa et al., 2012; Kono et al., 2014). Furthermore, the rate of CEF has been shown to increase during photosynthetic induction (Fan et al., 2007), which likely prevents PSI acceptor site limitation when the Calvin-Banson-Bassham cycle is not yet properly optimized. The pgr5 mutant under fluctuating light might thus suffer also due to defects in re-routing the electrons upon photosynthetic induction. However, it is not yet known whether the role of photosynthetic induction is as crucial upon shift from low light to high light, as it is known to be upon shift of plants from darkness to light. Noteworthy, the Arabidopsis ndh mutants do not show any phenotype under the fluctuating light conditions described above, indicating the $\mathrm{NDH}$ complex does not bear a crucial role for acclimation to light conditions in which light intensity fluctuates between low and moderately high light (Suorsa et al., 2012).

\section{Involvement of CEF Upon Drought and Extreme Temperatures}

Drought stress with subsequent lack of $\mathrm{CO}_{2}$ for carbon fixation due to stomatal closure is one of the well-characterized environmental conditions triggering CEF (Golding and Johnson, 2003; Golding et al., 2004; Rumeau et al., 2007; Munekage et al., 2008; Kohzuma et al., 2009; Johnson, 2011). Arabidopsis plants overexpressing PGR5 have been shown to exhibit enhanced tolerance to drought stress (Long et al., 2008). Furthermore, drought-stressed Arabidopsis plants upregulated the expression of the PGR5, PGRL1A, and PGRL1B genes with concomitant accumulation of the PGR5 and PGRL1 proteins, whereas both the transcript and the protein levels of the $\mathrm{NDH}$ subunit $\mathrm{NdhH}$ remained stable (Lehtimaki et al., 2010). On the other hand, under low air humidity but normal watering, the tobacco $n d h b$ mutant has been shown to exhibit compromised growth as compared to WT plants (Horvath et al., 2000). Additionally, tobacco ndhb mutants were shown to upregulate the PGR5PGRL1-dependent CEF under drought stress (Munne-Bosch et al., 2005), indicting compensatory roles for the two CEF routes.

It is pivotal to keep in mind that due to evolutionary adaptation to a variety of growth habitats, physiological responses to environmental stress conditions are highly species-dependent, and results obtained with one species cannot always be generalized to others. For instance, in comparison to upregulated levels of PGR5 upon drought stress in Arabidopsis (Long et al., 2008; Lehtimaki et al., 2010), Rosa meillandina, which is very tolerant against high temperatures and high light as long as there is no shortage of water, showed increased contents of PGR5 as a response to heat and light (Paredes and Quiles, 2013). However, combination of heat, high light intensity and drought stress induced decreased levels of PGR5 with simultaneous upregulation of the $\mathrm{NDH}$ complex and plastid terminal oxidase
(PTOX), strongly indicating a role for $\mathrm{NDH}$ in chlororespiration in $R$. meillandina under those conditions (Paredes and Quiles, 2013).

Similar to drought stress, also cold stress causes lowered carbon fixation, which in turn results in an excessive amount of reducing equivalents and thus imbalanced stromal redox state (Figure 1). Cold stress in combination with light illumination threatens particularly PSI (Sonoike and Terashima, 1994; Sonoike et al., 1995; Tjus et al., 1998; Kudoh and Sonoike, 2002), and similar to situation during fluctuating light, CEF plays an important role in protection of PSI also under low-temperaturecaused stress. For example, treatment of spinach leaves with low temperatures has been shown to enhance CEF (Kou et al., 2013). In addition, a 3-day-treatment of maize plants with lowered temperature induced upregulation of particularly the PGR-mediated CEF (Savitch et al., 2011). In line with this, coldacclimated Arabidopsis plants showed upregulation of PGR5PGRL1-dependent CEF, while NDH complex abundancies rather decreased upon cold acclimation (Ivanov et al., 2012). On the other hand, rice mutants lacking the NDH complex showed a growth defect as a response to lowered temperatures (Yamori et al., 2011). Furthermore, the tobacco $n d h b$ mutants exposed to a combination of low temperature and low light intensity showed disturbed regulation of electron transfer chain (ETC) as compared to WT ( $\mathrm{Li}$ et al., 2004). It seems likely that the responses against cold stress in chilling-sensitive plants differ from those of tolerant species, which again highlights the broad variety in CEF responses in different species. Enhanced CEF has also been suggested to be involved in drastic modulations of ETC occurring in conifer needles during winter (Oquist and Huner, 2003), yet experimental evidence is still needed to verify these hypothesis.

Root temperature has been shown to bear particular significance for the temperature responses in terms of CEF regulation. Visible damage and complete blockage of both LEF and CEF occurred in rice when only stem, but not root temperature was lowered (Suzuki et al., 2011). Furthermore, this condition has been shown to upregulate both $\mathrm{NDH}$ complex and the plastid terminal oxidase contents in a tropical plant Spathiphyllum wallisii, whereas upregulation of the PGR5PGRL1 - dependent CEF as well as that of the PGR5 levels were observed either when entire plant was chilled (Segura and Quiles, 2015), or when roots were chilled but stems heated (Soto et al., 2014).

\section{CEF Plays an Important Role under Early Developmental Phases}

Cyclic electron flow seems to play a crucial role under the early developmental stages. A recent comprehensive study on photosynthetic features of Arabidopsis seeds demonstrated that the light which the embryo chloroplasts receive is enriched in far red region of the spectrum, which preferentially excites PSI (Allorent et al., 2015). Consequently, green seeds were found to possess an increased CEF as compared to leaves, and the seed germination rates positively correlated with CEF activity 


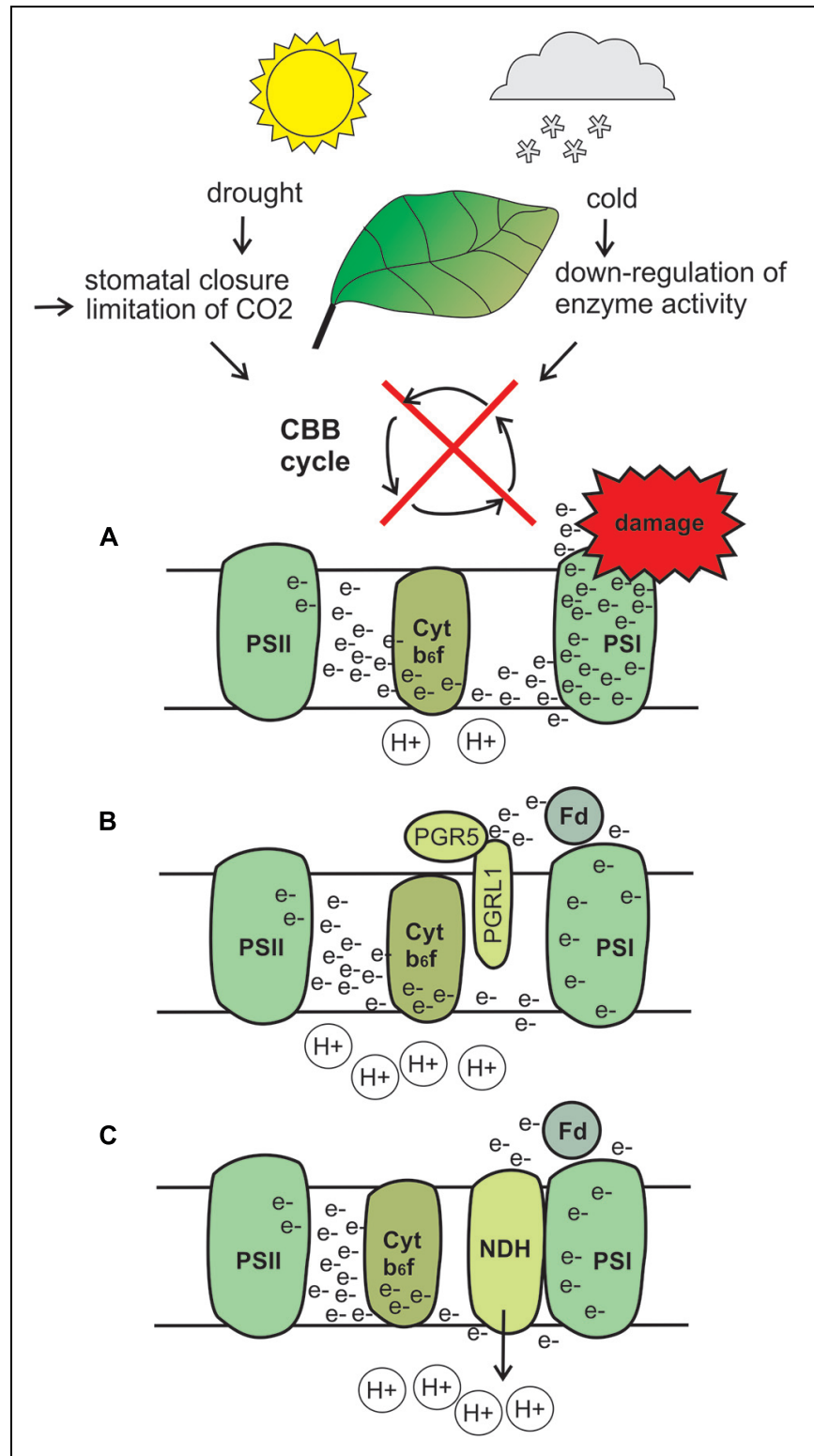

FIGURE 1 | Hypothetical model describing upregulation of cyclic electron flow (CEF) under drought and cold stress. Drought stress induces stomatal closure and subsequent limitation in $\mathrm{CO}_{2}$ levels, whereas cold stress slows down the enzyme activity. Both conditions result in downregulation of the Calvin-Benson-Bassham cycle. (A) A hypothetical situation in which CEF would not function, and which would thus end up in severe over-reduction of the ETC and stroma, and finally to photodamage. (B) When the PGR5 and PGRL1 proteins receive electrons from ferredoxin (Fd) and trigger lumen acidification, electron flow toward PSI becomes, thus preventing ETC and stromal components from over-reduction and photodamage. In addition, lumen acidification leads to induction of non-photochemical quenching (NPQ; not drawn to the figure). (C) The NDH complex receives electrons from $\mathrm{Fd}$, thus functioning as a safety valve for the excess of electrons. Furthermore, NDH likely functions as a proton pump, which participates in lumen acidification. Note that under most natural conditions, drought and cold likely induce upregulation of both CEF routes to some degree, but the preferential route depends on plant species. The illustrations describe only the hypothetical, not the actual position of the proteins and protein complexes. CBB, Calvin-Benson-Bassham cycle; PSI, photosystem; Fd, ferredoxin; e-, electron; $\mathrm{H}+$, proton.
(Allorent et al., 2015). Notably, even though in C3-leaves the proportion of the PGR5-PGRL1-dependent CEF is higher than that of the NDH-dependent CEF, in seeds the latter one plays a more prominent role (Allorent et al., 2015). The importance of the NDH-dependent CEF route under early developmental phases is in line with earlier reports showing that the NDHcomplex subunits are present and assembled as subcomplexes already in etioplasts (Kanervo et al., 2008), and the final assembly of the PSI-NDH supercomplex rapidly takes place upon exposure of etioplasts into light (Kanervo et al., 2008; Peng et al., 2009).

The enhanced CEF under early developmental stages highlights the proposed role of CEF in photoprotection of PSI. We have previously shown that photoprotection of PSI by the PGR5 protein under fluctuating light plays its most crucial role upon early developmental stage (Suorsa et al., 2012, 2013). Indeed, the synthesis of PSI is most active in young leaves, which causes strong decline in the contents of the PSI assembly factors upon leaf maturation (Krech et al., 2012; Liu et al., 2012). This corroborates with the idea of PSI being vulnerable and thus in the need of intensive protoprotection particularly under early developmental states.

The role of CEF during the opposite phase of leaf development, i.e., under senescence, still remains elusive. Similar to early developmental phases, leaf senescence involves oxidative stress. Early phases of the senescence are characterized by upregulation of the antioxidant machinery, which allows controlled remobilization and recycling of nutrients and photoassimilates to other parts of the plants (Juvany et al., 2013). Upon later senescence, declining antioxidant network induces massive oxidative stress, which leads to damage and ultimately to death. Intriguingly, it has been shown that tobacco plants lacking the chloroplast-encoded NDH subunit $\mathrm{NdhF}$, and thus containing only residual amounts of the $\mathrm{NDH}$ complex, exhibited delayed senescence under optimal greenhouse conditions (Zapata et al., 2005, 2007). Furthermore, the tobacco $\Delta n d h F$ mutants showed increased fitness, likely due to delayed senescence (Zapata et al., 2007). However, the exact molecular mechanism(s) behind the delayed senescence in the tobacco $\Delta n d h F$ mutant remain to be characterized, neither it is known whether the effect can be generalized to other long-lived plants.

\section{Concluding Remarks}

During the past few years, knowledge on the protein subunits and assembly factors involved in the two main routes for CEF has been substantially increasing. The available information about molecular mechanisms behind CEF is now enabling more intense research focus being directed toward physiological significance of CEF. There is already compelling evidence about the significance of particularly the NDH-dependent CEF for the energy metabolism in developing embryos. Both routes of CEF also seem to respond to stresses induced by drought or coldness. Furthermore, the PGR5 protein has been shown to have a crucial role in acclimation to fluctuating light conditions. However, results concerning CEF that have been acquired with one species 
cannot be necessarily generalized to cover all species. Thus, future studies with a wide range of evolutionary divergent species are still needed in order to obtain a more comprehensive view on the impact of CEF on plant development and environmental acclimation.

\section{References}

Allorent, G., Osorio, S., Vu, J. L., Falconet, D., Jouhet, J., Kuntz, M., et al. (2015). Adjustments of embryonic photosynthetic activity modulate seed fitness in Arabidopsis thaliana. New Phytol. 205, 707-719. doi: 10.1111/nph.13044

Armbruster, U., Labs, M., Pribil, M., Viola, S., Xu, W., Scharfenberg, M., et al. (2013). Arabidopsis CURVATURE THYLAKOID1 proteins modify thylakoid architecture by inducing membrane curvature. Plant Cell 25, 2661-2678. doi: 10.1105/tpc.113.113118

Avenson, T. J., Cruz, J. A., Kanazawa, A., and Kramer, D. M. (2005). Regulating the proton budget of higher plant photosynthesis. Proc. Natl. Acad. Sci. U.S.A. 102, 9709-9713. doi: 10.1073/pnas.0503952102

Baradaran, R., Berrisford, J. M., Minhas, G. S., and Sazanov, L. A. (2013). Crystal structure of the entire respiratory complex I. Nature 494, 443-448. doi: 10.1038 /nature 11871

Casano, L. M., Martin, M., and Sabater, B. (2001). Hydrogen peroxide mediates the induction of chloroplastic Ndh complex under photooxidative stress in barley. Plant Physiol. 125, 1450-1458. doi: 10.1104/pp.125.3.1450

DalCorso, G., Pesaresi, P., Masiero, S., Aseeva, E., Schunemann, D., Finazzi, G., et al. (2008). A complex containing PGRL1 and PGR5 is involved in the switch between linear and cyclic electron flow in Arabidopsis. Cell 132, 273-285. doi: 10.1016/j.cell.2007.12.028

Dang, K. V., Plet, J., Tolleter, D., Jokel, M., Cuine, S., Carrier, P., et al. (2014). Combined increases in mitochondrial cooperation and oxygen photoreduction compensate for deficiency in cyclic electron flow in Chlamydomonas reinhardtii. Plant Cell 26, 3036-3050. doi: 10.1105/tpc.114.1 26375

Desplats, C., Mus, F., Cuine, S., Billon, E., Cournac, L., and Peltier, G. (2009). Characterization of $\mathrm{Nda} 2$, a plastoquinone-reducing type II NAD(P)H dehydrogenase in Chlamydomonas chloroplasts. J. Biol. Chem. 284, 4148-4157. doi: 10.1074/jbc.M804546200

Fan, D. Y., Nie, Q., Hope, A. B., Hillier, W., Pogson, B. J., and Chow, W. S. (2007). Quantification of cyclic electron flow around Photosystem I in spinach leaves during photosynthetic induction. Photosynth. Res. 94, 347-357. doi: 10.1007/s11120-006-9127-z

Fan, X., Zhang, J., Li, W., and Peng, L. (2015). The NdhV subunit is required to stabilize the chloroplast NADH dehydrogenase-like complex in Arabidopsis. Plant J. 82, 221-231. doi: 10.1111/tpj.12807

Garcia-Andrade, J., Ramirez, V., Lopez, A., and Vera, P. (2013). Mediated plastid RNA editing in plant immunity. PLoS Pathog. 9:e1003713. doi: 10.1371/journal.ppat.1003713

Golding, A. J., Finazzi, G., and Johnson, G. N. (2004). Reduction of the thylakoid electron transport chain by stromal reductants-evidence for activation of cyclic electron transport upon dark adaptation or under drought. Planta 220, 356-363. doi: 10.1007/s00425-004-1345-z

Golding, A. J., and Johnson, G. N. (2003). Down-regulation of linear and activation of cyclic electron transport during drought. Planta 218, 107-114. doi: 10.1007/s00425-003-1077-5

Grieco, M., Suorsa, M., Jajoo, A., Tikkanen, M., and Aro, E. M. (2015). Lightharvesting II antenna trimers connect energetically the entire photosynthetic machinery - including both photosystems II and I. Biochim. Biophys. Acta 1847, 607-619. doi: 10.1016/j.bbabio.2015.03.004

Grieco, M., Tikkanen, M., Paakkarinen, V., Kangasjarvi, S., and Aro, E. M. (2012). Steady-state phosphorylation of light-harvesting complex II proteins preserves photosystem I under fluctuating white light. Plant Physiol. 160, 1896-1910. doi: 10.1104/pp.112.206466

Hertle, A. P., Blunder, T., Wunder, T., Pesaresi, P., Pribil, M., Armbruster, U., et al. (2013). PGRL1 is the elusive ferredoxin-plastoquinone reductase in photosynthetic cyclic electron flow. Mol. Cell 49, 511-523. doi: 10.1016/j.molcel.2012.11.030

\section{Acknowledgments}

Dr. Sari Järvi and Dr. Paolo Pesaresi are thanked for critical reading of the manuscript. Academy of Finland (projects 271832, 273870) and the ITN "Calipso" are acknowledged for funding.

Horvath, E. M., Peter, S. O., Joet, T., Rumeau, D., Cournac, L., Horvath, G. V., et al. (2000). Targeted inactivation of the plastid ndhB gene in tobacco results in an enhanced sensitivity of photosynthesis to moderate stomatal closure. Plant Physiol. 123, 1337-1350. doi: 10.1104/pp.123.4.1337

Ifuku, K., Endo, T., Shikanai, T., and Aro, E. M. (2011). Structure of the chloroplast NADH dehydrogenase-like complex: nomenclature for nuclearencoded subunits. Plant Cell Physiol. 52, 1560-1568. doi: 10.1093/pcp/pcr098

Ivanov, A. G., Rosso, D., Savitch, L. V., Stachula, P., Rosembert, M., Oquist, G., et al. (2012). Implications of alternative electron sinks in increased resistance of PSII and PSI photochemistry to high light stress in cold-acclimated Arabidopsis thaliana. Photosynth. Res. 113, 191-206. doi: 10.1007/s11120-012-9769-y

Iwai, M., Takizawa, K., Tokutsu, R., Okamuro, A., Takahashi, Y., and Minagawa, J. (2010). Isolation of the elusive supercomplex that drives cyclic electron flow in photosynthesis. Nature 464, 1210-1213. doi: 10.1038/nature08885

Jans, F., Mignolet, E., Houyoux, P. A., Cardol, P., Ghysels, B., Cuine, S., et al. (2008). A type II NAD(P)H dehydrogenase mediates light-independent plastoquinone reduction in the chloroplast of Chlamydomonas. Proc. Natl. Acad. Sci. U.S.A. 105, 20546-20551. doi: 10.1073/pnas.0806896105

Johnson, G. N. (2011). Physiology of PSI cyclic electron transport in higher plants. Biochim. Biophys. Acta 1807, 384-389. doi: 10.1016/j.bbabio.2010.11.009

Johnson, X., Steinbeck, J., Dent, R. M., Takahashi, H., Richaud, P., Ozawa, S., et al. (2014). Proton gradient regulation 5-mediated cyclic electron flow under ATP- or redox-limited conditions: a study of DeltaATpase pgr5 and DeltarbcL pgr5 mutants in the green alga Chlamydomonas reinhardtii. Plant Physiol. 165, 438-452. doi: 10.1104/pp.113.233593

Joliot, P., and Johnson, G. N. (2011). Regulation of cyclic and linear electron flow in higher plants. Proc. Natl. Acad. Sci. U.S.A. 108, 13317-13322. doi: 10.1073/pnas.1110189108

Juvany, M., Muller, M., and Munne-Bosch, S. (2013). Photo-oxidative stress in emerging and senescing leaves: a mirror image? J. Exp. Bot. 64, 3087-3098. doi: $10.1093 /$ jxb/ert174

Kanervo, E., Singh, M., Suorsa, M., Paakkarinen, V., Aro, E., Battchikova, N., et al. (2008). Expression of protein complexes and individual proteins upon transition of etioplasts to chloroplasts in pea (Pisum sativum). Plant Cell Physiol. 49, 396-410. doi: 10.1093/pcp/pcn016

Kohzuma, K., Cruz, J. A., Akashi, K., Hoshiyasu, S., Munekage, Y. N., Yokota, A., et al. (2009). The long-term responses of the photosynthetic proton circuit to drought. Plant Cell Environ. 32, 209-219. doi: 10.1111/j.13653040.2008.01912.x

Kono, M., Noguchi, K., and Terashima, I. (2014). Roles of the cyclic electron flow around PSI (CEF-PSI) and $\mathrm{O}(2)$-dependent alternative pathways in regulation of the photosynthetic electron flow in short-term fluctuating light in Arabidopsis thaliana. Plant Cell Physiol. 55, 990-1004. doi: 10.1093/pcp/ pcu033

Kou, J., Takahashi, S., Oguchi, R., Fan, D.-Y., Badger, M., and Chow, W. S. (2013). Estimation of the steady-state cyclic electron flux around PSI in spinach leaf discs in white light, CO2-enriched air and other varied conditions. Funct. Plant Biol. 40, 1018-1028.

Kouril, R., Strouhal, O., Nosek, L., Lenobel, R., Chamrad, I., Boekema, E. J., et al (2014). Structural characterization of a plant photosystem I and NAD(P)H dehydrogenase supercomplex. Plant J. 77, 568-576. doi: 10.1111/tpj.1 2402

Krech, K., Ruf, S., Masduki, F. F., Thiele, W., Bednarczyk, D., Albus, C. A., et al. (2012). The plastid genome-encoded ycf4 protein functions as a nonessential assembly factor for photosystem I in higher plants. Plant Physiol. 159, 579-591. doi: 10.1104/pp.112.196642

Kudoh, H., and Sonoike, K. (2002). Irreversible damage to photosystem I by chilling in the light: cause of the degradation of chlorophyll after returning to normal growth temperature. Planta 215, 541-548. doi: 10.1007/s00425-0020790-9 
Lehtimaki, N., Lintala, M., Allahverdiyeva, Y., Aro, E. M., and Mulo, P. (2010). Drought stress-induced upregulation of components involved in ferredoxindependent cyclic electron transfer. J. Plant Physiol. 167, 1018-1022. doi: 10.1016/j.jplph.2010.02.006

Leister, D., and Shikanai, T. (2013). Complexities and protein complexes in the antimycin A-sensitive pathway of cyclic electron flow in plants. Front. Plant Sci. 4:161. doi: $10.3389 /$ fpls.2013.00161

Li, X. G., Duan, W., Meng, Q. W., Zou, Q., and Zhao, S. J. (2004). The function of chloroplastic $\mathrm{NAD}(\mathrm{P}) \mathrm{H}$ dehydrogenase in tobacco during chilling stress under low irradiance. Plant Cell Physiol. 45, 103-108. doi: 10.1093/pcp/pch011

Li, Z., Wakao, S., Fischer, B. B., and Niyogi, K. K. (2009). Sensing and responding to excess light. Annu. Rev. Plant Biol. 60, 239-260. doi: 10.1146/annurev.arplant.58.032806.103844

Liu, J., Yang, H., Lu, Q., Wen, X., Chen, F., Peng, L., et al. (2012). PsbPdomain protein1, a nuclear-encoded thylakoid lumenal protein, is essential for photosystem I assembly in Arabidopsis. Plant Cell 24, 4992-5006. doi: 10.1105/tpc.112.106542

Long, T. A., Okegawa, Y., Shikanai, T., Schmidt, G. W., and Covert, S. F. (2008). Conserved role of proton gradient regulation 5 in the regulation of PSI cyclic electron transport. Planta 228, 907-918. doi: 10.1007/s00425-008-0789-y

Majeran, W., Zybailov, B., Ytterberg, A. J., Dunsmore, J., Sun, Q., and van Wijk, K. J. (2008). Consequences of C4 differentiation for chloroplast membrane proteomes in maize mesophyll and bundle sheath cells. Mol. Cell. Proteomics 7, 1609-1638. doi: 10.1074/mcp.M800016-MCP200

Maul, J. E., Lilly, J. W., Cui, L., dePamphilis, C. W., Miller, W., Harris, E. H., et al. (2002). The Chlamydomonas reinhardtii plastid chromosome: islands of genes in a sea of repeats. Plant Cell 14, 2659-2679. doi: 10.1105/tpc.0 06155

Munekage, Y., Hashimoto, M., Miyake, C., Tomizawa, K., Endo, T., Tasaka, M., et al. (2004). Cyclic electron flow around photosystem I is essential for photosynthesis. Nature 429, 579-582. doi: 10.1038/nature02598

Munekage, Y., Hojo, M., Meurer, J., Endo, T., Tasaka, M., and Shikanai, T. (2002). PGR5 is involved in cyclic electron flow around photosystem I and is essential for photoprotection in Arabidopsis. Cell 110, 361-371. doi: 10.1016/S00928674(02)00867-X

Munekage, Y. N., Genty, B., and Peltier, G. (2008). Effect of PGR5 impairment on photosynthesis and growth in Arabidopsis thaliana. Plant Cell Physiol. 49, 1688-1698. doi: 10.1093/pcp/pcn140

Munne-Bosch, S., Shikanai, T., and Asada, K. (2005). Enhanced ferredoxindependent cyclic electron flow around photosystem I and alpha-tocopherol quinone accumulation in water-stressed ndhB-inactivated tobacco mutants. Planta 222, 502-511. doi: 10.1007/s00425-005-1548-y

Mus, F., Cournac, L., Cardettini, V., Caruana, A., and Peltier, G. (2005). Inhibitor studies on non-photochemical plastoquinone reduction and $\mathrm{H}(2)$ photoproduction in Chlamydomonas reinhardtii. Biochim. Biophys. Acta 1708, 322-332. doi: 10.1016/j.bbabio.2005.05.003

Nishikawa, Y., Yamamoto, H., Okegawa, Y., Wada, S., Sato, N., Taira, Y., et al. (2012). PGR5-dependent cyclic electron transport around PSI contributes to the redox homeostasis in chloroplasts rather than $\mathrm{CO}(2)$ fixation and biomass production in rice. Plant Cell Physiol. 53, 2117-2126. doi: 10.1093/pcp/ pcs153

Oquist, G., and Huner, N. (2003). Photosynthesis of overwintering evergreen plants. Annu. Rev. Plant Biol. 54, 329-355. doi: 10.1146/annurev.arplant.54.072402.115741

Paredes, M., and Quiles, M. J. (2013). Stimulation of chlororespiration by drought under heat and high illumination in Rosa meillandina. J. Plant Physiol. 170, 165-171. doi: 10.1016/j.jplph.2012.09.010

Peng, L., Fukao, Y., Fujiwara, M., Takami, T., and Shikanai, T. (2009). Efficient operation of $\mathrm{NAD}(\mathrm{P}) \mathrm{H}$ dehydrogenase requires supercomplex formation with photosystem I via minor LHCI in Arabidopsis. Plant Cell 21, 3623-3640. doi: $10.1105 /$ tpc. 109.068791

Peng, L., and Shikanai, T. (2011). Supercomplex formation with photosystem I is required for the stabilization of the chloroplast NADH dehydrogenaselike complex in Arabidopsis. Plant Physiol. 155, 1629-1639. doi: 10.1104/pp.110.171264

Peng, L., Shimizu, H., and Shikanai, T. (2008). The chloroplast NAD(P)H dehydrogenase complex interacts with photosystem I in Arabidopsis. J. Biol. Chem. 283, 34873-34879. doi: 10.1074/jbc.M803207200
Peng, L., Yamamoto, H., and Shikanai, T. (2010). Structure and biogenesis of the chloroplast NAD(P)H dehydrogenase complex. Biochim. Biophys. Acta 1807, 945-953. doi: 10.1016/j.bbabio.2010.10.015

Petroutsos, D., Terauchi, A. M., Busch, A., Hirschmann, I., Merchant, S. S., Finazzi, G., et al. (2009). PGRL1 participates in iron-induced remodeling of the photosynthetic apparatus and in energy metabolism in Chlamydomonas reinhardtii. J. Biol. Chem. 284, 32770-32781. doi: 10.1074/jbc.M109.050468

Queval, G., and Foyer, C. H. (2012). Redox regulation of photosynthetic gene expression. Philos. Trans. R. Soc. Lond. B Biol. Sci. 367, 3475-3485. doi: 10.1098/rstb.2012.0068

Reiland, S., Finazzi, G., Endler, A., Willig, A., Baerenfaller, K., Grossmann, J., et al. (2011). Comparative phosphoproteome profiling reveals a function of the STN8 kinase in fine-tuning of cyclic electron flow (CEF). Proc. Natl. Acad. Sci. U.S.A. 108, 12955-12960. doi: 10.1073/pnas.1104734108

Rodriguez-Herva, J. J., Gonzalez-Melendi, P., Cuartas-Lanza, R., AntunezLamas, M., Rio-Alvarez, I., Li, Z., et al. (2012). A bacterial cysteine protease effector protein interferes with photosynthesis to suppress plant innate immune responses. Cell. Microbiol. 14, 669-681. doi: 10.1111/j.1462-5822.2012.0 1749.x

Rumeau, D., Peltier, G., and Cournac, L. (2007). Chlororespiration and cyclic electron flow around PSI during photosynthesis and plant stress response. Plant Cell Environ. 30, 1041-1051. doi: 10.1111/j.1365-3040.2007.0 1675.x

Savitch, L. V., Ivanov, A. G., Gudynaite-Savitch, L., Huner, N. P., and Simmonds, J. (2011). Cold stress effects on PSI photochemistry in Zea mays: differential increase of FQR-dependent cyclic electron flow and functional implications. Plant Cell Physiol. 52, 1042-1054. doi: 10.1093/pcp/pcr056

Segura, M. V., and Quiles, M. J. (2015). Involvement of chlororespiration in chilling stress in the tropical species Spathiphyllum wallisii. Plant Cell Environ. 38, 525-533. doi: 10.1111/pce.12406

Shikanai, T. (2014). Central role of cyclic electron transport around photosystem I in the regulation of photosynthesis. Curr. Opin. Biotechnol. 26, 25-30. doi: 10.1016/j.copbio.2013.08.012

Sirpio, S., Allahverdiyeva, Y., Holmstrom, M., Khrouchtchova, A., Haldrup, A., Battchikova, N., et al. (2009). Novel nuclear-encoded subunits of the chloroplast $\mathrm{NAD}(\mathrm{P}) \mathrm{H}$ dehydrogenase complex. J. Biol. Chem. 284, 905-912. doi: $10.1074 /$ jbc.M805404200

Sonoike, K., and Terashima, I. (1994). Mechanism of photosystem-i photoinhibition in leaves of Cucumis-sativus L. Planta 194, 287-293. doi: $10.1007 / \mathrm{BF} 01101690$

Sonoike, K., Terashima, I., Iwaki, M., and Itoh, S. (1995). Destruction of photosystem I iron-sulfur centers in leaves of Cucumis sativus L. by weak illumination at chilling temperatures. FEBS Lett. 362, 235-238. doi: 10.1016/0014-5793(95)00254-7

Soto, A., Hernandez, L., and Quiles, M. J. (2014). High root temperature affects the tolerance to high light intensity in Spathiphyllum plants. Plant Sci. 227, 84-89. doi: 10.1016/j.plantsci.2014.07.004

Strand, D. D., Livingston, A. K., Satoh-Cruz, M., Froehlich, J. E., Maurino, V. G., and Kramer, D. M. (2015). Activation of cyclic electron flow by hydrogen peroxide in vivo. Proc. Natl. Acad. Sci. U.S.A. 112, 5539-5544. doi: $10.1073 /$ pnas. 1418223112

Suorsa, M., Grieco, M., Jarvi, S., Gollan, P. J., Kangasjarvi, S., Tikkanen, M., et al. (2013). PGR5 ensures photosynthetic control to safeguard photosystem I under fluctuating light conditions. Plant Signal. Behav. 8, e22741. doi: $10.4161 /$ psb. 22741

Suorsa, M., Järvi, S., Grieco, M., Nurmi, M., Pietrzykowska, M., Rantala, M., et al. (2012). PROTONGRADIENTREGULATION5 is essential for proper acclimation of Arabidopsis photosystem I to naturally and artificially fluctuating light conditions. Plant Cell 24, 2934-2948. doi: 10.1105/tpc.112.0 97162

Suorsa, M., Sirpio, S., Paakkarinen, V., Kumari, N., Holmstrom, M., and Aro, E. M. (2010). Two proteins homologous to PsbQ are novel subunits of the chloroplast NAD(P)H dehydrogenase. Plant Cell Physiol. 51, 877-883. doi: $10.1093 / \mathrm{pcp} / \mathrm{pcq} 070$

Suzuki, K., Ohmori, Y., and Ratel, E. (2011). High root temperature blocks both linear and cyclic electron transport in the dark during chilling of the leaves of rice seedlings. Plant Cell Physiol. 52, 1697-1707. doi: 10.1093/pcp/ pcr104 
Tikkanen, M., Grieco, M., Kangasjarvi, S., and Aro, E. M. (2010). Thylakoid protein phosphorylation in higher plant chloroplasts optimizes electron transfer under fluctuating light. Plant Physiol. 152, 723-735. doi: 10.1104/pp.109.150250

Tikkanen, M., Rantala, S., and Aro, E. M. (2015). Electron flow from PSII to PSI under high light is controlled by PGR5 but not by PSBS. Front. Plant Sci. 6:521. doi: 10.3389/fpls.2015.00521

Tjus, S. E., Moller, B. L., and Scheller, H. V. (1998). Photosystem I is an early target of photoinhibition in barley illuminated at chilling temperatures. Plant Physiol. 116, 755-764. doi: 10.1104/pp.116.2.755

Tolleter, D., Ghysels, B., Alric, J., Petroutsos, D., Tolstygina, I., Krawietz, D., et al. (2011). Control of hydrogen photoproduction by the proton gradient generated by cyclic electron flow in Chlamydomonas reinhardtii. Plant Cell 23, 2619-2630. doi: 10.1105/tpc.111.086876

Ueda, M., Kuniyoshi, T., Yamamoto, H., Sugimoto, K., Ishizaki, K., Kohchi, T., et al. (2012). Composition and physiological function of the chloroplast NADH dehydrogenase-like complex in Marchantia polymorpha. Plant J. 72, 683-693. doi: 10.1111/j.1365-313X.2012.05115.X

Wakasugi, T., Tsudzuki, J., Ito, S., Nakashima, K., Tsudzuki, T., and Sugiura, M. (1994). Loss of all ndh genes as determined by sequencing the entire chloroplast genome of the black pine Pinus thunbergii. Proc. Natl. Acad. Sci. U.S.A. 91, 9794-9798. doi: 10.1073/pnas.91.21.9794

Wang, C., Yamamoto, H., and Shikanai, T. (2014). Role of cyclic electron transport around photosystem I in regulating proton motive force. Biochim. Biophys. Acta. 1847, 931-938. doi: 10.1016/j.bbabio.2014.11.013

Yabuta, S., Ifuku, K., Takabayashi, A., Ishihara, S., Ido, K., Ishikawa, N., et al. (2010). Three PsbQ-like proteins are required for the function of the chloroplast NAD(P)H dehydrogenase complex in Arabidopsis. Plant Cell Physiol. 51, 866876. doi: $10.1093 /$ pcp/pcq060

Yamamoto, H., Peng, L., Fukao, Y., and Shikanai, T. (2011). An Src homology 3 domain-like fold protein forms a ferredoxin binding site for the chloroplast NADH dehydrogenase-like complex in Arabidopsis. Plant Cell 23, 1480-1493. doi: $10.1105 /$ tpc. 110.080291
Yamamoto, H., and Shikanai, T. (2013). In planta mutagenesis of Src homology 3 domain-like fold of $\mathrm{NdhS}$, a ferredoxin-binding subunit of the chloroplast NADH dehydrogenase-like complex in Arabidopsis: a conserved Arg-193 plays a critical role in ferredoxin binding. J. Biol. Chem. 288, 36328-36337. doi: 10.1074/jbc.M113.511584

Yamori, W., Sakata, N., Suzuki, Y., Shikanai, T., and Makino, A. (2011). Cyclic electron flow around photosystem I via chloroplast $\mathrm{NAD}(\mathrm{P}) \mathrm{H}$ dehydrogenase (NDH) complex performs a significant physiological role during photosynthesis and plant growth at low temperature in rice. Plant J. 68, 966-976. doi: 10.1111/j.1365-313X.2011.04747.x

Yamori, W., Shikanai, T., and Makino, A. (2015). Photosystem I cyclic electron flow via chloroplast NADH dehydrogenease-like complex performs a physiological role for photosynthesis at low light. Sci. Rep. 5, 13908. doi: 10.1038/srep13908

Zapata, J. M., Gasulla, F., Esteban-Carrasco, A., Barreno, E., and Guera, A. (2007). Inactivation of a plastid evolutionary conserved gene affects PSII electron transport, life span and fitness of tobacco plants. New Phytol. 174, 357-366. doi: 10.1111/j.1469-8137.2007.02001.x

Zapata, J. M., Guera, A., Esteban-Carrasco, A., Martin, M., and Sabater, B. (2005) Chloroplasts regulate leaf senescence: delayed senescence in transgenic ndhFdefective tobacco. Cell Death Differ. 12, 1277-1284. doi: 10.1038/sj.cdd.44 01657

Conflict of Interest Statement: The author declares that the research was conducted in the absence of any commercial or financial relationships that could be construed as a potential conflict of interest.

Copyright (c) 2015 Suorsa. This is an open-access article distributed under the terms of the Creative Commons Attribution License (CC BY). The use, distribution or reproduction in other forums is permitted, provided the original author(s) or licensor are credited and that the original publication in this journal is cited, in accordance with accepted academic practice. No use, distribution or reproduction is permitted which does not comply with these terms. 